\title{
Beam-beam effects in RHIC
}

\author{
Y. Luo, M. Bai, W. Fischer, \\ C. Montag, S. White
}

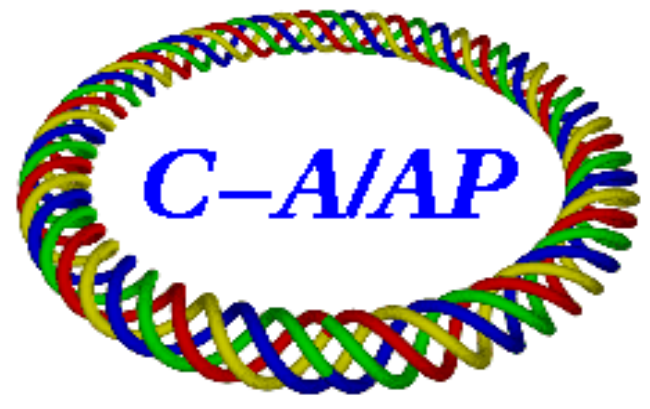

Collider-Accelerator Department

Brookhaven National Laboratory

Upton, NY 11973

Notice: This document has been authorized by employees of Brookhaven Science Associates, LLC under Contract No. DE-AC02-98CH10886 with the U.S. Department of Energy. The United States Government retains a nonexclusive, paid-up, irrevocable, world-wide license to publish or reproduce the published form of this document, or allow others to do so, for United States Government purposes. 


\title{
BEAM-BEAM EFFECTS IN RHIC*
}

\author{
Y. Luo, M. Bai, W. Fischer, C. Montag, S. White, \\ Brookhaven National Laboratory, Upton, NY 11973, USA
}

\section{Abstract}

In this article we review the beam-beam effects in the polarized proton runs in the Relativistic Heavy Ion Collider (RHIC). The operational observations, limitations, and the next luminosity goal are presented and discussed. With an upgraded polarized proton source, the proton bunch intensity will be increased from $1.7 \times 10^{11}$ up to $3.0 \times 10^{11}$. To accommodate the large beam-beam tune spread in the current tune space between $2 / 3$ and 7/10 and to compensate the nonlinear beam-beam resonance driving terms, head-on beam-beam compensation with electron lenses (e-lenses) is to be installed.

\section{INTRODUCTION}

RHIC consists of two superconducting rings, the Blue ring and the Yellow ring. They intersect at 6 locations around the ring circumference. The two beams collide at two interaction points (IPs), IPI6 and IP8. Fig. 1 shows the layout of RHIC. RHIC is capable of colliding heavy ions and polarized protons ( $p-p)$. The maximum total beambeam parameter for 2 IPs was 0.003 in the $100 \mathrm{GeV} \mathrm{Au-}$ $\mathrm{Au}$ collision and 0.017 in the $250 \mathrm{GeV}$ p-p collision. In this article we only discuss the beam-beam effects in the $\mathrm{p}-\mathrm{p}$ runs.

The main limitation to the beam lifetime in the RHIC p$\mathrm{p}$ runs are the beam-beam interactions, the nonlinear magnetic field errors in the interaction regions (IRs), the nonlinear chromaticities with low $\beta^{*}$ s and the machine and beam parameter modulations.

The working point in the RHIC p-p runs is chosen to provide a good beam-beam lifetime and to maintain the proton polarization during the energy ramp and physics stores. The nominal working point $\left(Q_{x}, Q_{y}\right)=(28.695,29.685)$ is constrained between $2 / 3$ and $7 / 10$. $7 / 10$ is a 10th order betatron resonance and also a spin depolarization resonance. Experiments and simulations have shown that the beam lifetime and the proton polarization are reduced when the vertical tune of the proton beam is close to $7 / 10$ [1].

Figure 2 shows the proton tune footprint including beambeam interactions. The proton bunch intensity is $2.0 \times 10^{11}$ and the $95 \%$ normalized transverse emittance (6 times the rms normalized emittance $\left.\epsilon_{\mathrm{n}, \mathrm{rms}}\right)$ is $15 \pi \mathrm{mm}$.mrad leading to a beam-beam parameter of 0.02. From Fig. 2, there is not enough tune space to hold the large beam-beam tune spread when the proton bunch intensity is larger than

* This work was supported by Brookhaven Science Associates, LLC under Contract No. DE-AC02-98CH10886 with the U.S. Department of Energy.

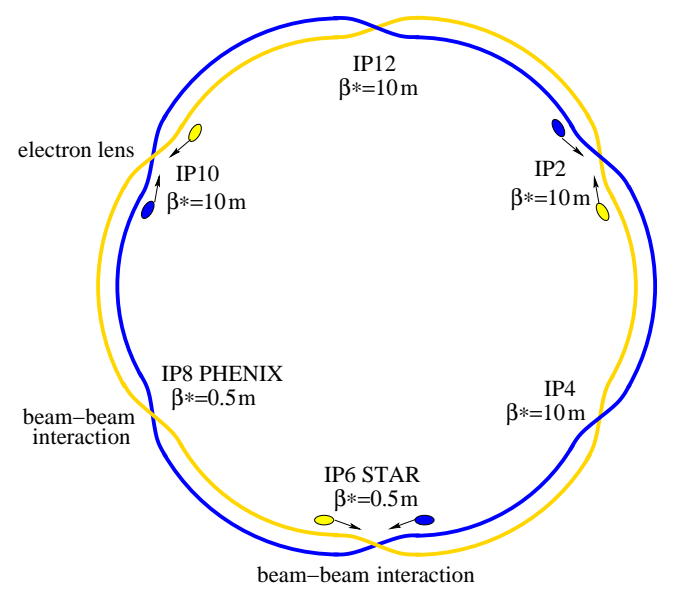

Figure 1: Layout of RHIC. Two beams collide at IP6 and IP8. The e-lenses are to be installed close to IP10.

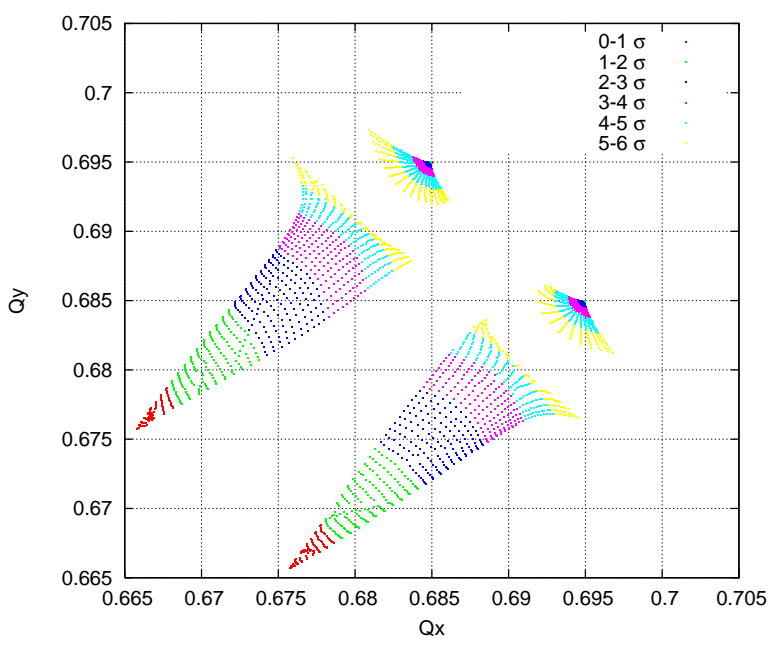

Figure 2: Tune footprints without and with beam-beam. In this calculation, the bunch intensity is $2.0 \times 10^{11}$.

$2.0 \times 10^{11}$ [2]. Simulations and experiments have been continuously carried out to explore new tune spaces.

\section{OBSERVATIONS}

\section{Previous $p$ - $p$ runs}

The luminosity in the $\mathrm{p}-\mathrm{p}$ collision is given by

$$
L=\frac{N_{\mathrm{p}}^{2} N_{\mathrm{b}} \gamma f_{\mathrm{rev}}}{4 \pi \epsilon_{\mathrm{n}, \mathrm{rms}} \beta^{*}} H\left(\frac{\beta^{*}}{\sigma_{\mathrm{l}}}\right) .
$$

Here $N_{\mathrm{p}}$ is the proton bunch intensity, and $N_{\mathrm{b}}$ the number of bunches, $\gamma$ the Lorentz factor, $f_{\text {rev }}$ the revolution frequency. $\epsilon_{\mathrm{n}, \mathrm{rms}}$ is the rms normalized emittance and $\sigma_{\mathrm{l}}$ the 
rms bunch length. $H\left(\frac{\beta^{*}}{\sigma_{1}}\right)$ is the luminosity reduction factor due to hour-glass effect.

The total beam-beam parameter, or the linear incoherent beam-beam tune shift is

$$
\xi=\frac{N_{\mathrm{p}} r_{\mathrm{p}}}{2 \pi \epsilon_{\mathrm{n}, \mathrm{rms}}} .
$$

Here $r_{\mathrm{p}}$ is the classical radius of proton. We assumed 2 collisions at IP6 and IP8.

In the 2009 RHIC $100 \mathrm{GeV}$ p-p run, with $\beta^{*}=0.7 \mathrm{~m}$ and a bunch intensity of $1.5 \times 10^{11}$, we observed a shorter beam lifetime of 7 hours compared to the 12 hours in the 2008 RHIC $100 \mathrm{GeV}$ p-p run with $\beta^{*}=1.0 \mathrm{~m}[3,4,5,6]$. In the 2012 RHIC $100 \mathrm{GeV}$ p-p run, $\beta^{*}=0.85 \mathrm{~m}$ lattices were adopted, the beam lifetime was 16 hours with a typical bunch intensity of $1.65 \times 10^{11}$ [7].

In the 2011 and $2012250 \mathrm{GeV}$ p-p runs a common $9 \mathrm{MHz}$ RF system was used to produce a long bunch length to maintain both transverse and longitudinal emittances during the energy ramp [8,7]. When the beams reached store energy, re-bucketing with $28 \mathrm{MHz}$ RF systems was used to achieve a shorter bunch length. In the $2012250 \mathrm{GeV}$ p-p run, $\beta^{*}$ was $0.65 \mathrm{~m}$ and the maximum bunch intensity reached $1.7 \times 10^{11}$. The typical store length was 8 hours. During this run, several techniques were tried to reduce the ramp loss due to the large longitudinal emittance to increase bunch intensity [7].

\section{Beam Lifetime}

During RHIC p-p runs, beam losses at store are dominated by the beam-beam interactions. In the 2011 and 2012 $250 \mathrm{GeV}$ run, the beam lifetime was 50-100 hours without collisions, depending on machine tuning. After the beams were brought into collision, we observed a large beam loss during the first 1-2 hours and a small beam loss during the rest of the store [9]. Empirically, the beam losses can be parameterized with double exponentials,

$$
N_{\mathrm{p}}(t)=A_{1} \exp \left(-t / \tau_{1}\right)+A_{2} \exp \left(-t / \tau_{2}\right),
$$

where $N_{p}(t)$ is the bunch intensity, and $A_{1,2}$ and $\tau_{1,2}$ are fitting parameters.

Figure 3 shows an example of typical bunch intensity evolutions in the $250 \mathrm{GeV}$ p-p run. In RHIC, few bunches out of 109 bunches have only one collision instead of two collisions. Fitting with Eq. (1), for bunches with 1 collision, $\left(\tau_{1}, \tau_{2}\right)$ are 1.5 hours, 100 hours). While for bunches with 2 collisions, they are ( 0.8 hour, 30 hours). We are investigating the losses on a shorter time scale, which are given by $\tau_{1}$.

\section{Transverse Emittance and Bunch Length}

During the $2012250 \mathrm{GeV}$ p-p run, the normalized 95\% transverse emittances were of the order of $15 \pi \mathrm{mm} . \mathrm{mrad}$ at the beginning of stores. We did not observe clear transverse emittance growth during the store. The emittances

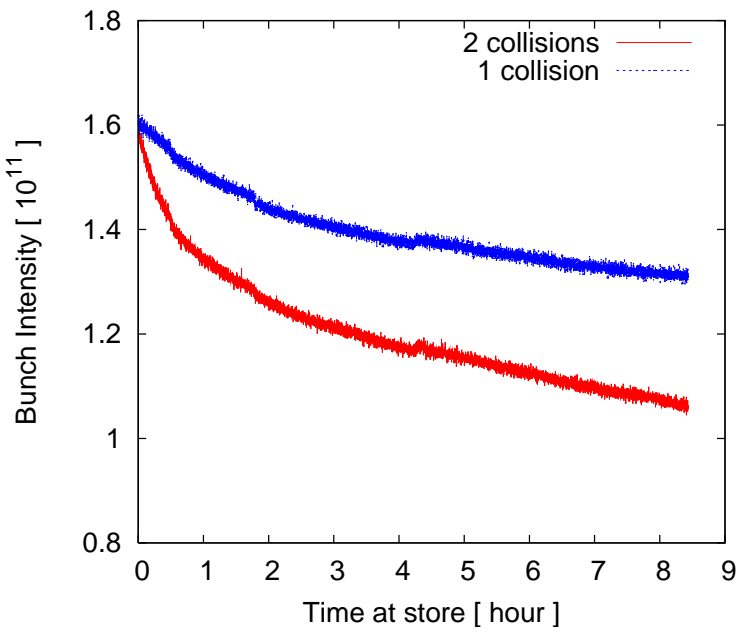

Figure 3: Typical evolutions of bunch intensities with 1 and 2 collisions at store in $2011250 \mathrm{GeV}$ p-p run. The fill number is 15386 .

were measured with an ionization profile monitor (IPM) and also derived from luminosity. All the particle loss happened in the transverse planes, which was due to the limited transverse dynamic aperture.

Bunch length was measured with a wall current monitor (WCM). During the $2012250 \mathrm{GeV}$ p-p run, the measured bunch lengthening during the stores was less than $20 \%$. Analytical calculation shows that the intrabeam scattering (IBS) effect contributes less than $10 \%$ of the total bunch lengthening. Longitudinal instabilities were observed at store. A Landau cavity of $197 \mathrm{MHz}$ with $200 \mathrm{kV}$ voltages was used to overcome it $[7,8]$.

\section{Coherent Beam-beam}

At RHIC the coherent beam-beam modes are routinely observed with the beam transfer function (BTF) using the phase-lock-loop (PLL) tune meter [10]. By scanning the excitation frequency one can measure the amplitude and phase responses to this frequency. Fig. 3 shows an example of BTF measurements taken during a store with 12 bunches colliding at the two IPs during the $2012100 \mathrm{GeV}$ p-p run. In this Figure the $\pi$-mode is missing in the horizontal plane, which could be explained by a transfer of the Landau damping from one plane to the other [11].

Dedicated beam experiments were carried out in the 2012 p-p runs to study the effects of low order resonances on coherent beam-beam modes and possible suppression techniques [11]. In one experiment, we deliberately moved the tunes of the Blue ring toward $Q_{y}=2 / 3$ resonance. We measured the $\pi$-mode located at 0.669 . Beam losses were seen only in the Blue ring. No transverse emittance blowup was observed. We concluded that the beam loss in the Blue ring was due to an incoherent effect. Otherwise, the $\pi$-modes could have interfered with the $Q_{y}=2 / 3$ resonance and affected both beams.

To eliminate the coherent beam-beam modes, we put the 


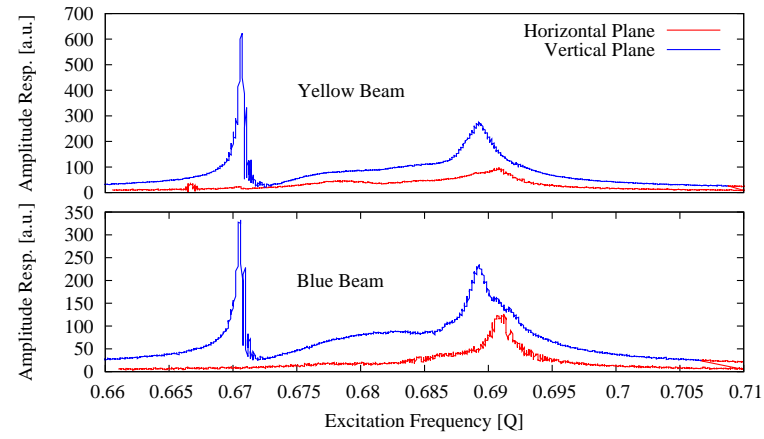

Figure 4: BTF measurements for 12 bunches colliding in 2 IPs at $100 \mathrm{GeV}$.

tunes of the two beams farther apart than the beam-beam parameter to decouple the beams. We tried several physics stores in the $20122050 \mathrm{GeV}$ p-p run with beams colliding at working points $\left(Q_{x}, Q_{y}\right)=(0.695,0.685)$ in the Blue ring and $(0.72,0.73)$ in the Yellow ring. A strong emittance blow-up was observed in three fills out of four as soon as the beams were brought into collision. The polarization decay of the beam in the Yellow ring was $2.1 \%$ /hour, which was higher than $1.4 \%$ /hour at the nominal working point [7].

\section{LIMITATIONS}

\section{Low $\beta^{*}$ lattices}

In order to further increase the luminosity, we can either increase the bunch intensity or reduce $\beta^{*}$. We will discuss the bunch intensity increase later. A low $\beta^{*}$ lattice increases the $\beta$ functions in the triplet quadrupoles and therefore the particles will sample large nonlinear magnetic field errors at these locations. As a result, the dynamic aperture will be reduced [12]. For example, in the $2009100 \mathrm{GeV}$ pp run, we used a lattice with $\beta^{*}=0.7 \mathrm{~m}$ which gave a short beam lifetime [6]. At $250 \mathrm{GeV}$, we achieved $\beta^{*}=0.65 \mathrm{~m}$. The reason is that the transverse beam size is smaller at $250 \mathrm{GeV}$ than at $100 \mathrm{GeV}$ [13].

A low $\beta^{*}$ lattice also increases the nonlinear chromaticity and reduces the off-momentum dynamic aperture. Chromatic analysis shows that the nonlinear chromaticities are mostly originating from the low $\beta$ insertions IR6 and IR8 [14]. The nonlinear chromaticities increase dramatically with the decreased $\beta^{*}$. Fig. 5 shows the calculated second and third order chromaticities as functions of $\beta^{*}$. Large second order chromaticities push the particles with large momentum errors to the 3 rd or 10th order resonances. Several correction techniques of nonlinear chromaticities have been tested and implemented in RHIC [15]. To further reduce $\beta^{*}$, we need to balance the hour-glass effect, beam lifetime reduction and the luminosity gain.

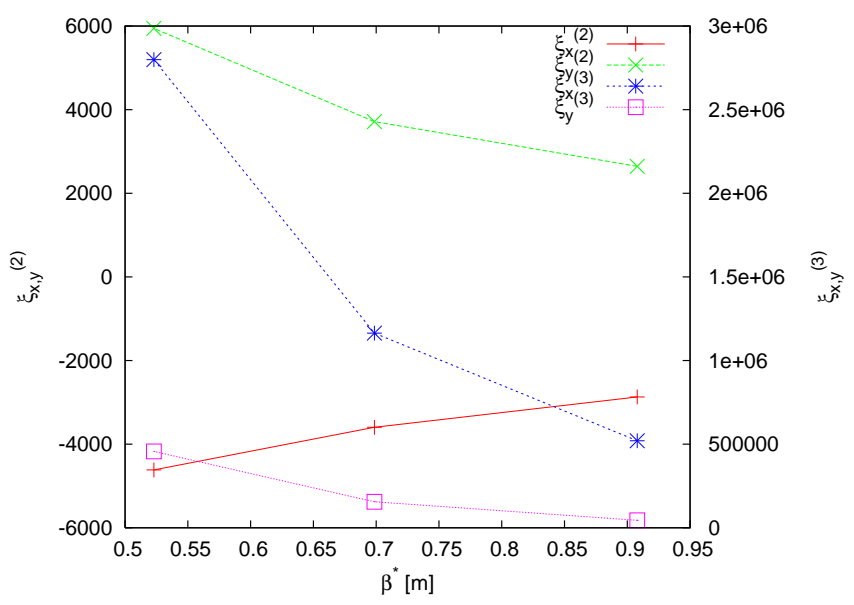

Figure 5: Calculated second and third order chromaticities vs. $\beta^{*}$ in the p-p runs.

\section{$3 Q_{x, y}$ Resonances}

To mitigate the coupling between two beams, we would like to mirror the working points of the two RHIC rings on both sides of the diagonal in the tune space. However, in the operation we had to operate with both working points below the diagonal for better beam lifetimes. It was understood in the $2006100 \mathrm{GeV}$ p-p run that the strong $3 Q_{x}$ resonances at $Q_{x}=2 / 3$ prevented the working point above the diagonal [4]. At that time, the proton bunch intensity was $1.3 \times 10^{11}$.

In the $2012100 \mathrm{GeV}$ run, even with both working points below the diagonal, when the bunch intensity was higher than $1.7 \times 10^{11}$, we observed a larger beam loss due to the $3 Q_{y}$ resonance which is located at $Q_{y}=2 / 3$ [7]. The main contributions to the third order resonances are from the sextupole and skew sextupole components in the IR6 and IR8. To reduce the resonance stop-bands, we routinely correct the local sextupole and skew sextuopole errors with IR orbit bumps by minimizing the feed-down tune shifts [16], which improved the beam losses experimentally. Measurement and correction of the global third order resonance driving terms with $\mathrm{AC}$ dipole excitation were also applied [17, 18].

\section{$10 \mathrm{~Hz}$ Orbit Oscillation}

In the beginning of the $2008100 \mathrm{GeV}$ p-p run, we tested a near-integer working point $(0.96,0.95)$ in the Blue ring while keeping the working point in the Yellow ring at $(0.695,0.685)$. Weak-strong beam-beam simulation shows that there is a wider tune space with good dynamic apertures than the working point $(0.695,0.685)$ [19]. The spin simulation shows that there are weaker spin depolarization resonances in this region as well.

However, operating at near-integer tunes turned out very challenging [5]. With such tunes, we found that it was 
difficult to correct the closed orbit and to control the $\beta$ beat. Moreover, both detectors reported high background rates from the beam in the Blue ring when two beams were brought into collision. These backgrounds were caused by horizontal orbit vibrations around $10 \mathrm{~Hz}$ which originated from mechanical vibrations of the low- $\beta$ triplets driven by the cryogenic flow [20]. To correct the $10 \mathrm{~Hz}$ orbit oscillations, we developed a local $10 \mathrm{~Hz}$ orbit feedback system and succeeded in the 2011 p-p run [21]. With the feedback on, the amplitude of the $10 \mathrm{~Hz}$ orbit oscillation is reduced from 3000 microns down to 250 microns peak-to-peak in the triplet quadrupoles. We plan to re-visit the near-integer working point in future beam experiment sessions.

\section{E-LENS PROJECT}

\section{Increasing bunch intensity}

To further increase the luminosity in the polarized proton operation in RHIC, we plan to increase the proton bunch intensity from currently $1.7 \times 10^{11}$ up to $3.0 \times 10^{11}$ with an upgraded polarized proton source [22]. However, with such a high proton bunch intensity, the total beam-beam tune shift and the amplitude dependent beam-beam tune spread will be about 0.03 . To accomodate this large beam-beam tune spread in the current tune space and to compensate the large beam-beam resonance driving terms, we decided to install head-on beam-beam compensation in RHIC [2].

High proton bunch intensity may cause radiation issues, BPM cable heating, resistive wall heating, electron cloud instability and so on [23]. The present Accelerator Safety Envelope (ASE) imposes a strict intensity limitation. For 109 bunches at $250 \mathrm{GeV}$, the maximum allowed bunch intensity is $2.3 \times 10^{11}$. To increase bunch intensity above that, we need put more shieldings in various places. In 2012, during dedicated beam experiments, we injected 109 bunches with averaged bunch intensity $2.6 \times 10^{11}$. A helium temperature rise of about $\Delta T=40 \mathrm{mK}$ was observed, which agrees with predictions.

\section{Head-on Beam-beam Compensation}

Head-on beam-beam compensation in proton colliders with electron beams was first proposed and studied by Tsyganov in 1993 [24]. The idea is to introduce a low energy electron beam to collide head-on with the opposing proton beam to compensate the beam-beam nonlinear effects. The device which provides the electron beam is called an electron lens (e-lens). A pulsed elens had been successfully installed and operated in the Tevatron to compensate the long-range beam-beam tune shifts [25, 26, 27, 28].

In our current design for the RHIC head-on beam-beam compensation, two e-lenses are needed [29, 30], one for the Blue ring and one for the Yellow ring. They will be installed on either side of the interaction point IP10 where the two proton beams are separated in the vertical plane. To stabilize the electron beam, each compensation region is surrounded by a superconducting solenoid. The magnetic field of the solenoids ranges from $3 \mathrm{~T}$ to $6 \mathrm{~T}$. To cancel their effect on the global betatron coupling and spin dynamics for the polarized proton beams, the two solenoids are powered with opposite polarities.

Each RHIC e-lens system consists of a DC electron gun, an electron beam transport to the main solenoid, a superconducting main solenoid in which the interaction with the proton beam occurs, an electron transport to the collector, and an electron collector. The main solenoid, the electron gun and collector and the electron beam transport have been designed and fabricated in the past 2-3 years [31, 32, 33]. This summer, a test bench including a superconducting solenoid has been set up to measure the electron beam parameters and test the beam instrumentation [34]. We plan to install one e-lens in the Blue ring before the $2013250 \mathrm{GeV}$ p-p run. Lattices for half head-on beam-beam compensation have been developed.

With partial beam-beam compensation, we expect a factor of two improvement in luminosity. Fig. 6 shows the calculated dynamic aperture under different beam-beam conditions. Here half beam-beam compensation (HBBC) compensates half of the total beam-beam parameter. For better beam-beam resonance cancellation, we adjust the phase advances between IP8 and the e-lens to be $k \pi$. Simulations show that the $k \pi$ phase advances and global second order chromaticity correction improve the dynamic aperture of half beam-beam compensation [2].

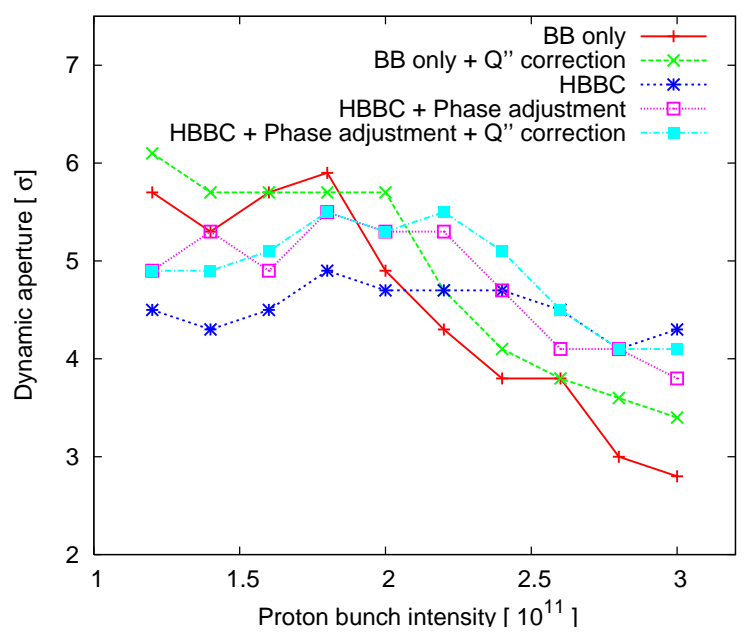

Figure 6: Dynamic apertures of half head-on beam-beam compensation vs. bunch intensity.

\section{SIMULATIONS}

Beam-beam simulations have been playing a crucial role at RHIC to understand the current beam-beam observations and to predict the effects of head-on beam-beam compensation. Both weak-strong and strong-strong simulation codes had been developed in recent years. With the weakstrong beam-beam interaction model, we calculate the fre- 
quency map, action diffusion, and $10^{6}$ turns dynamic aperture [35]. Dynamic aperture has been frequently used to judge the stability of lattices and the effects of beam-beam interaction on RHIC dynamic aperture. With the strongstrong model, we calculate the beam spectrum, beam transfer function, and beam emittance blow-up [36].

\section{Weak-Strong Model}

Our simulation code for the weak-strong beam-beam simulation is SimTrack, which is a C++ library for the optics calculation and particle tracking for high energy hadron accelerators [37]. In this code, the magnetic elements are treated using 4th order symplectic integration. 4-D and 6-D weak-strong beam-beam treatments are included. Particles are tracked element by element. To save computing time in the long-term tracking, multipoles are treated as thin lenses.

We also perform multi-particle tracking with the weakstrong model to calculate beam lifetime and emittance growth. The challenge here is to obtain meaningful physics results with limited computing resources and computing time. Considering that the particles with large amplitudes in the bunch tail are likely to be lost in the tracking and to overcome the statistical error in the calculated particle loss rate and to better represent the particles in the tail of a 6-D Gaussian bunch, we track particles with an initial hollow Gaussian distribution [38].

\section{Strong-strong Model}

The multi-particle simulations for RHIC are performed with the tracking code BeamBeam3D [39, 36]. In this code, the particle motions between IPs are represented by 4-D linear matrices. The tune changes due to linear and nonlinear chromaticities are included. The nonlinear magnetic errors in the IRs are applied at IPs instead of in the IRs, but with a $\pi / 2$ phase advance. To calculate the beambeam kicks, the beam distribution and the field are computed every turn from the macro-particle distribution. This model allows for a realistic approach which includes Landau damping and gives the correct frequency of the beambeam coherent modes.

\section{SUMMARY}

In this article we reviewed the beam-beam observations in the polarized proton runs in RHIC. The limitations from the tune space, low $\beta^{*}$ lattices, $3 Q_{x, y}$ resonances, and $10 \mathrm{~Hz}$ orbit modulations were discussed. For the next luminosity goal, head-on beam-beam compensation has been adopted to reduce the beam-beam tune spread and beambeam resonance driving terms. Together with the upgraded polarized proton source, we expect to double the luminosity. We summaried the beam-beam simulation codes and methods for the RHIC beam-beam studies.

\section{REFERENCES}

[1] M. Bai, et al., Phys. Rev. Lett. 96, 174801 (2006)

[2] Y. Luo, W. Fischer, et al., Phys. Rev. ST Accel. Beams 15, 051004 (2012).

[3] M. Bai, et al., TUODKI04, Proceedings of PAC'2007.

[4] V. Ptitsyn, et al., MOPLS024, Proceedings of EPAC'2006.

[5] C. Montag, et al., WEPP018, Proceedings of EPAC'2008.

[6] C. Montag, et al., MOPEC033, Proceedings of IPAC'2010.

[7] V. Schoefer, et al., MOPPC25, Proceedings of IPAC'2012.

[8] H. Huang, et al., TUPZ035, Proceedings of IPAC'2011.

[9] W. Fischer, M. Bai, et al., BNL C-AD AP Note 235, 2006.

[10] W. Fischer, et al., TUPAS094, Proceedings of PAC'2007.

[11] S. White, et al., MOPPC028, Proceedings of IPAC'2012.

[12] Y. Luo, et al., WE6PFP007, Proceedings of PAC'2009.

[13] Y. Luo, et al., BNL C-AD AP Note 235, 2006.

[14] Y. Luo, et al., THP059, Proceedings of PAC'2011.

[15] Y. Luo, et al., WE6PFP006,Proceedings of PAC'2009.

[16] F. Pilat, Y. Luo, et al., WOAC007, Proceedings of PAC05.

[17] R. Tomas, et al., Phys. Rev. ST Accel. Beams 8, 024001

[18] Y. Luo, J. Bengtsson, et al., FRPMS109, Proceedings of PAC'2007.

[19] C. Montag, et al., TUPAS099, Proceedings of EPAC'2008.

[20] C. Montag, et al., Nucl. Instr. Meth. A 564, 26-31(2006).

[21] R. Michnoff, et al., MOP268, Proceedings of PAC'2011.

[22] A. Zelenski, Rev. Sci. Instrum. 81, 02B308 (2010).

[23] C. Montag, et al., WEPPR018, Proceedings of IPAC'2012.

[24] E. Tsyganov, et al., SSCL-Preprint-519, 1993.

[25] V. Shiltsev, et al., Phys. Rev. ST Accel. Beams 2, 071001 (1999).

[26] V. Shiltsev, et al., Phys. Rev. Lett. 99, 244801 (2007).

[27] V. Shiltsev, et al., Phys. Rev. ST Accel. Beams 11, 103501 (2008).

[28] X. Zhang, et al., Phys. Rev. ST Accel. Beams 11, 051002 (2008).

[29] Y. Luo and W. Fischer, BNL C-AD AP Note 286, July 2007.

[30] W. Fischer, Y. Luo, and A. Pikin (editors), RHIC Electron Lenses Design Report, BNL C-AD AP Note 359, 2009.

[31] W. Fischer, et al., MOPEC026, Proceedings of IPAC'2010.

[32] W. Fischer, et al., THP055, Proceedings of PAC'2011.

[33] W. Fischer, et al., WEOBA01, Proceedings of IPAC'2012.

[34] X. Gu, et al., WEPPD084, Proceedings of IPAC'2012.

[35] Y. Luo, W. Fischer, Beam Dynamics Newslette, No. 52, 2010.

[36] S. White, et al., MOPPC026, Proceedings of IPAC'2012.

[37] Y. Luo, TUPEC082, Proceedings of IPAC'2010.

[38] Y. Luo, W. Fischer, WEO2C02,Proceedings of HB2010.

[39] J. Qiang, Phys. Rev. ST Accel. Beams 9, 044204, 2006. 